\title{
Motion Estimation of a Moving Range Sensor by Image Sequences and Distorted Range Data
}

\author{
Atsuhiko Banno, Kazuhide Hasegawa and Katsushi Ikeuchi \\ Institute of Industrial Science \\ University of Tokyo \\ 4-6-1 Komaba, Meguro-ku, Tokyo 153-8505 Japan \\ \{vanno, k-hase, ki\}@cvl.iis.u-tokyo.ac.jp
}

\begin{abstract}
For a large scale object, scanning from the air is one of the most efficient methods of obtaining 3D data. In the case of large cultural heritage objects, there are some difficulties in scanning them with respect to safety and efficiency. To remedy these problems, we have been developing a novel 3D measurement system, the Floating Laser Range Sensor (FLRS) , in which a rage sensor is suspended beneath a balloon. The obtained data, however, have some distortion due to the intrascanning movement. In this paper, we propose a method to recover 3D range data obtained by a moving laser range sensor; this method is applicable not only to our FLRS, but also to a general moving range sensor. Using image sequences from a video camera mounted on the FLRS enables us to estimate the motion of the FLRS without any physical sensors such as gyros and GPS. At first, the initial values of camera motion parameters are estimated by perspective factorization. The next stage refines camera motion parameters using the relationships between camera images and the range data distortion. Finally, by using the refined parameter, the distorted range data are recovered. We applied this method to an actual scanning project and the results showed the effectiveness of our method.
\end{abstract}

Index Terms - Structure from Motion, Moving Range Sensor, Floating Laser Range Sensor, Perspective-Factorization

\section{INTRODUCTION}

Nowadays, many researches on real object modeling are making great progress because of the availability of accurate geometric data from three dimensional laser sensors. The techniques of real object modeling contribute toward numerous applications in wide areas such as academic investigation, industrial management, and entertainment.

Among them, one of the most important and comprehensive applications is modeling cultural heritage objects. Modeling these heritage objects has great significance in many aspects. Modeling them leads to digital archives of the object shapes. Utilizing these data enables us to restore the heritage objects' original shapes, even if the objects have been destroyed due to natural weathering, fire, disasters and wars. In addition, we can provide images of these objects through the Internet to people in their homes or in their offices. Thus, the techniques of real object modeling are available for many applications.

We have been conducting some projects to model large scale cultural heritage objects such as great Buddhas, historical buildings and suburban landscapes. Basically, to scan these large objects, a laser range finder is usually used with a tripod positioned on stable locations. In the case of scanning a large scale object, however, it often occurs that some part of the object is not visible from the laser range sensor on the ground. In spite of such a difficulty, we have scanned large objects from scaffolds temporally constructed nearby the object. However, this scaffold method requires costly, tedious construction time. In addition, it may be impossible to scan some parts of the object due to the limitation of available space for scaffold-building.

We are now conducting a project to model the Bayon Temple in Cambodia [7]; the temple's scale is about $100 \mathrm{x}$ 100 square meters. Scanning such a huge scale object from several scaffolds is unrealistic. To overcome this problem, several methods have been proposed. For example, aerial 3D measurements can be obtained by using a laser range sensor installed on a helicopter platform [12]. High frequency vibration of the platform, however, should be considered to ensure that we obtain highly accurate results. To avoid irrevocable destruction, the use of heavy equipment such as a crane should be eschewed when scanning a cultural heritage object.

Based upon the above considerations, we proposed a novel 3D measurement system, a Floating Laser Range Sensor (FLRS) [4]. This system digitizes large scale objects from the air while suspended from the underside of a balloon platform (Fig.1). Our balloon platform is certainly free from high frequency vibration such as that of a helicopter engine. The obtained range data are, however, distorted because the laser range sensor itself is moving during the scanning processes.

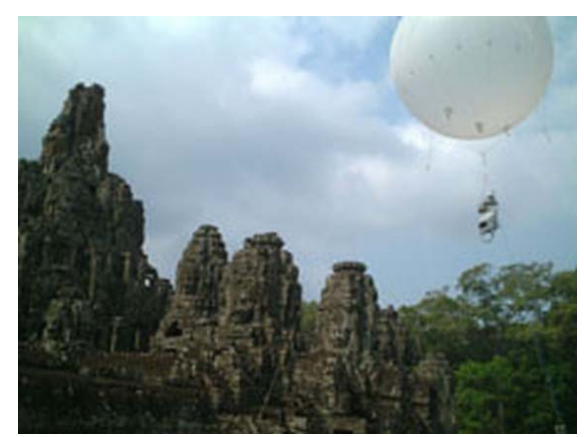

Fig. 1. Floating Laser Range Sensor and Bayon temple 
In this paper, we propose a method to recover $3 \mathrm{D}$ range data obtained by a moving laser range sensor. Not only is this method limited to the case of our FLRS, but it is also applicable to a general moving range sensor. We obtain distorted range data by using the range sensor, and obtain image sequences simultaneously by using the video camera mounted on the FLRS. Then the motion of the FLRS is estimated by the obtained images and distorted data without any physical sensors such as gyros and GPS. We estimate the camera motion parameters imposing some constraints, which include information derived from the distorted range data itself. In order to solve the non-linear optimization problem, we utilize a perspective factorization method as the initial value to avoid local minimums. Then, using the refined camera motion parameter, the distorted range data are recovered.

This paper organized as follows. In Section II, we briefly explain the perspective factorization, which is utilized as the initial value for the camera motion. In Section III, we describe our proposed algorithm for refinement of the parameters. In Section IV, we describe how we use this algorithm to model and recover the shape of the Bayon Temple in Cambodia. To evaluate our method, the recovered shapes are compared with other data obtained by a range sensor on the ground. Finally, we present our conclusions and summarize our possible future works.

\section{Perspective Factorization}

Estimations of the shape of an object or of camera motion by using images are called "Shape from Motion" or "Shape from Structure", and are main research fields in computer vision.

The factorization method proposed by Tomasi and Kanade [13] is one of the most effective algorithms for simultaneously recovering the shape of an object and the motion of the camera from an image sequence. This method was originally limited to the orthographic model. Then, however, the factorization was extended to several perspective approximations and applications [2][8][9][3]. Han and Kanade [3] proposed a factorization method with a perspective camera model. Using the weak-perspective projection model, they iteratively estimated the shape and the camera motion under the perspective model.

First, we briefly explain weak-perspective factorization, which is subsequently extended to the perspective factorization. The solution by the perspective factorization is utilized as the initial value for the optimizing problem described in Section III.

\section{A. Weak-Perspective Factorization}

Given a sequence of $\mathrm{F}$ images, in which we have tracked $\mathrm{P}$ interest points over all frames, each interest point $\mathrm{p}$ corresponds to a single point $\overrightarrow{s_{p}}$ on the object. In image coordinates, the trajectories of each interest point are denoted as $\left\{\left(u_{f p}, v_{f p}\right) \mid f=1, \ldots, F, p=1, \ldots, P 2 F \geq P\right\}$.
Using the horizontal coordinates $u_{f p}$, we can define an $F \times P$ matrix $U$. Each column of the matrix contains the horizontal coordinates of a single point in the frame order, while each row contains the horizontal coordinates for a single frame. Similarly, we can define an $F \times P$ matrix $V$ from the vertical coordinates $v_{f p}$.

The combined matrix of $2 F \times P$ becomes the measurement matrix as follow.

$$
W=\left(\frac{U}{V}\right)
$$

Each frame $\mathrm{f}$ is taken at camera position $\overrightarrow{t_{f}}$ in the world coordinates. The camera pose is described by the orthonormal unit vectors $\overrightarrow{i_{f}}, \overrightarrow{j_{f}}$ and $\overrightarrow{k_{f}}$. The vectors $\overrightarrow{i_{f}}$ and $\overrightarrow{j_{f}}$ correspond to the $\mathrm{x}$ and $\mathrm{y}$ axes of the camera coordinates, while the vector $\overrightarrow{k_{f}}$ corresponds to the $\mathrm{z}$ axis along the direction perpendicular to the image plane (Fig.2).

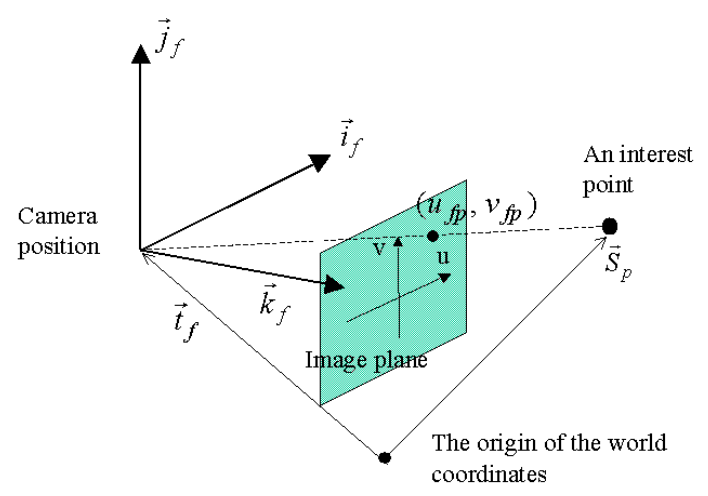

Fig. 2. the coordinate system: $\overrightarrow{t_{f}}$ denotes the position of the camera at time of frame $\mathrm{f}$. The camera pose is determined by three unit basis vectors.

Under the weak-perspective camera model, a single point in the world coordinates $\overrightarrow{s_{p}}$ is projected onto the image plane $\mathrm{f}$ as $\left(u_{f p}, v_{f p}\right)$.

$$
\begin{array}{r}
u_{f p}=\frac{f}{z_{f}} \overrightarrow{i_{f}}\left(\overrightarrow{s_{p}}-\overrightarrow{t_{f}}\right) \\
v_{f p}=\frac{f}{z_{f}} \overrightarrow{j_{f}}\left(\overrightarrow{s_{p}}-\overrightarrow{t_{f}}\right) \\
z_{f}=\overrightarrow{k_{f}}\left(\vec{c}-\overrightarrow{t_{f}}\right)
\end{array}
$$

The vector $\vec{c}$ is the center of mass of all interest points. Without loss of generality, the origin of the world coordinates can be placed at the centroid, that is $\vec{c}=0$. Then this means that $z_{f}=-\overrightarrow{k_{f}} \overrightarrow{t_{f}}$ to simplify the expansion of the following formulation. To summarize,

$$
\begin{gathered}
u_{f p}=\overrightarrow{m_{f}} \overrightarrow{s_{p}}+\mathbf{x}_{\mathbf{f}} \\
v_{f p}=\overrightarrow{n_{f}} \overrightarrow{s_{p}}+\mathbf{y}_{\mathbf{f}} \\
\text { where } \overrightarrow{m_{f}}=\frac{f}{z_{f}} \overrightarrow{i_{f}}, \quad \mathbf{x}_{\mathbf{f}}=-\frac{f}{z_{f}} \overrightarrow{i_{f}} \overrightarrow{t_{f}} \\
\overrightarrow{n_{f}}=\frac{f}{z_{f}} \overrightarrow{j_{f}}, \quad \mathbf{y}_{\mathbf{f}}=-\frac{f}{z_{f}} \overrightarrow{j_{f}} \overrightarrow{t_{f}}
\end{gathered}
$$


To be expressed this equation in a matrix form:

$$
\begin{aligned}
& \left(\begin{array}{ccc}
u_{11} & \ldots & u_{1 P} \\
u_{21} & \ldots & u_{2 P} \\
\vdots & \vdots & \vdots \\
u_{F 1} & \ldots & u_{F P} \\
v_{11} & \ldots & v_{1 P} \\
\vdots & \vdots & \vdots \\
v_{F 1} & \ldots & v_{F P}
\end{array}\right)=\left(\begin{array}{c}
\overrightarrow{m_{1}}{ }^{t} \\
\overrightarrow{m_{2}}{ }^{t} \\
\vdots \\
\overrightarrow{m_{F}}{ }^{t} \\
\overrightarrow{n_{1}} \\
\vdots \\
\overrightarrow{n_{F}}{ }^{t}
\end{array}\right)\left(\overrightarrow{s_{1}} \ldots \overrightarrow{s_{P}}\right) \\
& +\left(\begin{array}{c}
\mathbf{x}_{\mathbf{1}} \\
\mathbf{x}_{\mathbf{2}} \\
\vdots \\
\mathbf{x}_{\mathbf{F}} \\
\mathbf{y}_{\mathbf{1}} \\
\vdots \\
\mathbf{y}_{\mathbf{F}}
\end{array}\right)\left(\begin{array}{lll}
1 & \ldots & \\
\end{array}\right)
\end{aligned}
$$

Using that the center of all interest points is the origin,

$$
\sum_{p=1}^{P} u_{f p}=\sum_{p=1}^{P} \overrightarrow{m_{f}} \overrightarrow{s_{p}}+\sum_{p=1}^{P} \mathbf{x}_{\mathbf{f}}=P \mathbf{x}_{\mathbf{f}}
$$

similarly,

$$
\sum_{p=1}^{P} v_{f p}=P \mathbf{y}_{\mathbf{f}}
$$

We obtain the registered measurement matrix $\tilde{W}$, after translation $\tilde{W}=W-\left(\begin{array}{lllllll}\mathbf{x}_{\mathbf{1}} & \mathbf{x}_{\mathbf{2}} & \ldots & \mathbf{x}_{\mathbf{F}} & \mathbf{y}_{\mathbf{1}} & \ldots & \mathbf{y}_{\mathbf{F}}\end{array}\right)^{t}\left(\begin{array}{llll}1 & 1 & \ldots & \ldots\end{array}\right)$ as a product of two matrixes $M$ and $S$.

$$
\tilde{W}=M \cdot S
$$

where $M$ is a $2 F \times 3$ Matrix and $S$ is a $3 \times P$ Matrix.

The above decomposition, however, is not unique because any invertible $3 \times 3$ matrix $\mathrm{A}$ makes a valid decomposition of $\hat{W}$ as

$$
(M A)\left(A^{-1} S\right)=M\left(A A^{-1}\right) S=M S=\tilde{W}
$$

To get rid of this ambiguity, using the fact that the matrix $M$ represents the axes of the camera coordinates, the following constraints should be satisfied.

$$
\left|\vec{m}_{f}\right|=\left|\overrightarrow{n_{f}}\right|, \quad \overrightarrow{m_{f}} \cdot \overrightarrow{n_{f}}=0
$$

These constraints give us the motion matrix $M$ and the shape matrix $S$.

\section{B. Extension to Full-Perspective Factorization}

The above formulation is under the weak perspective camera model, which is a linear approximation of the perspective model. Next, using an iterative framework, we obtain approximate solutions under the non-linear perspective camera model.

Under the perspective camera model, the projective equation between the object point $\overrightarrow{s_{p}}$ in $3 \mathrm{D}$ world and the image coordinate $\left(u_{f p}, v_{f p}\right)$ is written as

$$
\begin{aligned}
& u_{f p}=f \frac{\overrightarrow{i_{f}}\left(\overrightarrow{s_{p}}-\overrightarrow{t_{f}}\right)}{\overrightarrow{k_{f}}\left(\overrightarrow{s_{p}}-\overrightarrow{t_{f}}\right)} \\
& v_{f p}=f \frac{\overrightarrow{j_{f}}\left(\overrightarrow{s_{p}}-\overrightarrow{t_{f}}\right)}{\overrightarrow{k_{f}}\left(\overrightarrow{s_{p}}-\overrightarrow{t_{f}}\right)}
\end{aligned}
$$

displacing $z_{f}=-\overrightarrow{k_{f}} \overrightarrow{t_{f}}$, we obtain the following equation.

$$
\begin{array}{r}
\left(\lambda_{f p}+1\right) u_{f p}=\frac{f}{z_{f}} \overrightarrow{i_{f}}\left(\overrightarrow{s_{p}}-\overrightarrow{t_{f}}\right) \\
\left(\lambda_{f p}+1\right) v_{f p}=\frac{f}{z_{f}} \overrightarrow{j_{f}}\left(\overrightarrow{s_{p}}-\overrightarrow{t_{f}}\right) \\
\text { where } \lambda_{f p}=\frac{\overrightarrow{k_{f}} \cdot \overrightarrow{s_{p}}}{z_{f}}
\end{array}
$$

Note that the right hand sides are the same form under the weak-perspective model (see the Eq. 2 and 3). This means, multiplying a image coordinate $\left(u_{f p}, v_{f p}\right)$ by a real number $\lambda_{f p}$ changes coordinates under the perspective model into coordinates under the weak-perspective model. Solving the value of $\lambda_{f p}$ iteratively, we can obtain motion parameters and coordinates of interest points under the perspective model in the framework of weak-perspective factorization.

The entire algorithm of the perspective factorization is as follows:

Input: An image sequence of $\mathrm{F}$ frames tracking $\mathrm{P}$ interest points.

Output:The positions of $\mathrm{P}$ interest points $\overrightarrow{s_{p}}$. The camera position $\overrightarrow{t_{f}}$ and poses $\overrightarrow{i_{f}}, \overrightarrow{j_{f}}, \overrightarrow{k_{f}}$ at each frame $\mathrm{f}$.

1) giving $\lambda_{f p}=0$

2) supposing the equations 17 and 18 , solve $\overrightarrow{s_{p}}, \overrightarrow{t_{f}}, \overrightarrow{i_{f}}, \overrightarrow{j_{f}}$, $\overrightarrow{k_{f}}$ and $z_{f}$ using the weak perspective factorization.

3) calculate $\lambda_{f p}$ by the equation 19 .

4) substituting $\lambda_{f p}$ into step2; repeat the above procedure until $\lambda_{f p}$ 's are close to the previous iteration.

\section{REFINEMENT OF CAMERA Motion}

Without noise in input, the above factorization method leads to the fine solution. As a result, recovered 3D shape through the estimated camera parameters is valid. Real images, however, contain a bit of noise. Therefore, it is not sufficient to recover range data obtained by the FLRS only through the factorization. For the sake of more refined estimation of camera parameters, we impose three constraints tracking, movement, and range data. Refined camera motion can be found through the minimization of a global functional. To minimize the function, the solution by the perspective factorization is utilized as the initial value to avoid local minimums.

\section{A. Constraint A (Tracking constraint)}

As the most fundamental constraint, any interest point $\overrightarrow{s_{p}}$ must be projected on each image plane at the coordinates $\left(u_{f p}, v_{f p}\right)$. This constraint conducts the following function:

$$
\begin{aligned}
F_{A}=\sum_{f=1}^{F} \sum_{p=1}^{P} & \left(\left(u_{f p}-f \frac{\overrightarrow{i_{f}}\left(\overrightarrow{s_{p}}-\overrightarrow{t_{f}}\right)}{\overrightarrow{k_{f}}\left(\overrightarrow{s_{p}}-\overrightarrow{t_{f}}\right)}\right)^{2}\right. \\
& \left.+\left(v_{f p}-f \frac{\overrightarrow{j_{f}}\left(\overrightarrow{s_{p}}-\overrightarrow{t_{f}}\right)}{\overrightarrow{k_{f}}\left(\overrightarrow{s_{p}}-\overrightarrow{t_{f}}\right)}\right)^{2}\right)
\end{aligned}
$$

The minimization of $F_{A}$ leads to the correct tracking of fixed interest points by a moving camera. However, we can 
see that the presence of parameters we are trying to estimate in the denominator makes this equation a difficult one. Then, suppose that instead, we consider the following function:

$$
\begin{array}{r}
F_{A}=\sum_{f=1}^{F} \sum_{p=1}^{P}\left(\left(\overrightarrow{k_{f}}\left(\overrightarrow{s_{p}}-\overrightarrow{t_{f}}\right) u_{f p}-f \overrightarrow{i_{f}}\left(\overrightarrow{s_{p}}-\overrightarrow{t_{f}}\right)\right)^{2}\right. \\
\left.+\left(\overrightarrow{k_{f}}\left(\overrightarrow{s_{p}}-\overrightarrow{t_{f}}\right) v_{f p}-f \overrightarrow{j_{f}}\left(\overrightarrow{s_{p}}-\overrightarrow{t_{f}}\right)\right)^{2}\right)
\end{array}
$$

\section{B. Constraint B (Movement constraint)}

One of the most significant reasons for adopting a balloon platform is to be free from high frequency vibration differently from a helicopter platform. In other words, a balloon platform is only under the influence of low frequency. The balloon of our FLRS is held with some wires swayed only by wind. This means that the movement of the balloon is expected to be smooth. Certainly, the movement of the balloon is free from rapid acceleration, rapid deceleration or acute course changing. Taking this fact into account, we consider following function:

$$
F_{B}=\int\left(w_{1}\left(\frac{\partial^{2} \overrightarrow{t_{f}}}{\partial t^{2}}\right)^{2}+w_{2}\left(\frac{\partial^{2} \mathbf{q}_{f}}{\partial t^{2}}\right)^{2}\right) d t
$$

Here, $\overrightarrow{t_{f}}$ denotes the position of the camera; $t$ is time; $w_{1}, w_{2}$ are weighted coefficients; and $\mathbf{q}_{f}$ is a unit quaternion which represents the rotation of camera pose. The presentation by quaternion is obtained immediately by $\overrightarrow{i_{f}}, \overrightarrow{j_{f}}$ and $\overrightarrow{k_{f}}$. The first term of the above integrand represents smoothness with respect to the camera's translation while the second represents smoothness with respect to the camera's rotation. When the motion of the camera is smooth, the function $F_{B}$ takes small value.

\section{Constraint $C$ (Range Data constraint)}

Taking a broad view of range data obtained by the FLRS, the data are distorted by the swing of the camera. We can find, however, that these data contain precise information locally; that information is utilized for refinement of the camera motion.

The laser range sensor re-radiates laser beams in raster scan order. This leads that we can instantly obtain the time when any pixel in the range image is scanned. If the video camera coincides with the range sensor, we can find the just frame among the sequence when the pixel is scanned. With the video camera calibrated with the range sensor, we can obtain the image coordinate of each interest point in the $3 \mathrm{D}$ world, in addition to the distance from the sensor to it once and for all. This constraint is very important since knowing the distances eliminates ambiguity with respect to scaling. The techniques of "Shape from Motion" or "Structure from Motion" only by images remain ambiguous about scaling. On the other hand, our method is able to derive some precise information about scale even from the distorted data and remove the ambiguity.
Then, we can conduct the third constraint to be minimized as follows:

$$
F_{C}=\sum_{p=1}^{P}\left\|\mathbf{x}_{f p}-\left(\overrightarrow{s_{p}}-t_{f p}\right)\right\|^{2}
$$

Here, the index $f p$ denotes the frame number when the range sensor scans the interest point $\mathrm{p}$, and the measured distance by the range sensor at this moment denotes $\mathbf{x}_{f p}$.

As $\mathbf{x}_{f p}=\left(x_{f p}, y_{f p}, z_{f p}\right)$, the above function can be rewritten to satisfy more rigorous constraint:

$$
\begin{aligned}
& F_{C}=\sum_{p=1}^{P}\left(\left(x_{f p}-\overrightarrow{i_{f p}}\left(\overrightarrow{s_{p}}-t_{f p}\right)\right)^{2}\right. \\
& \left.\quad+\left(y_{f p}-\overrightarrow{j_{f p}}\left(\overrightarrow{s_{p}}-\overrightarrow{t_{f p}}\right)\right)^{2}+\left(z_{f p}-\overrightarrow{k_{f p}}\left(\overrightarrow{s_{p}}-t_{f p}\right)\right)^{2}\right)
\end{aligned}
$$

\section{Global constraint}

The weighted sum

$$
F=w_{A} F_{A}+w_{B} F_{B}+w_{C} F_{C}
$$

leads to a global function. To minimize this function, we employ the Fletcher-Reeves method and the Polak-Ribiere method [10][5][11], which are types of the conjugate gradient method. Then, we use the golden section search to determine the magnitude of gradient directions. As mentioned in the previous sections, we input the solution by the perspective factorization as the initial value. Minimizing the function $F$ is basically quite difficult because this function has many local minimums. By employing the solution of the factorization as a fairly good approximation, we try to avoid them.

\section{EXPERIMENTS ON THE BAYON TEMPLE}

\section{A. Tracking}

In our system, we obtain 72 frames at a single scanning process. For interest point tracking, we use the SIFT key [6], which is robust for scaling, that is a movement along the view direction. A simple window matching method around the points traces the trajectory of each point throughout all the frames. After this procedure, we can derive about one hundred interest points from a sequence of 72 frames.

\section{B. Evaluation of the Recovered Shape}

To evaluate the accuracy of our shape recovery algorithm, we compare the recovered shape with other data, which are obtained by a range finder, the Cyrax 2500, positioned on the ground. Aligning two data by using ICP algorithm [1][15], we analyze the overlapped area. The result is shown in Figure 3. The blue fine data in both images is a non-distorted data (the correct data) obtained by the Cyrax 2500. The white coarse data in the middle figure indicates the original output data obtained by the FLRS, while the pink data in the lower figure indicates the data recovered by our method. One can easily find that the recovered $3 \mathrm{D}$ shape is well-fitted onto the Cyrax2500's data. In particular, taking notice of the area of ellipses in the middle figure, makes it obvious that our algorithm is effective. In the upper figure, the cross section, 


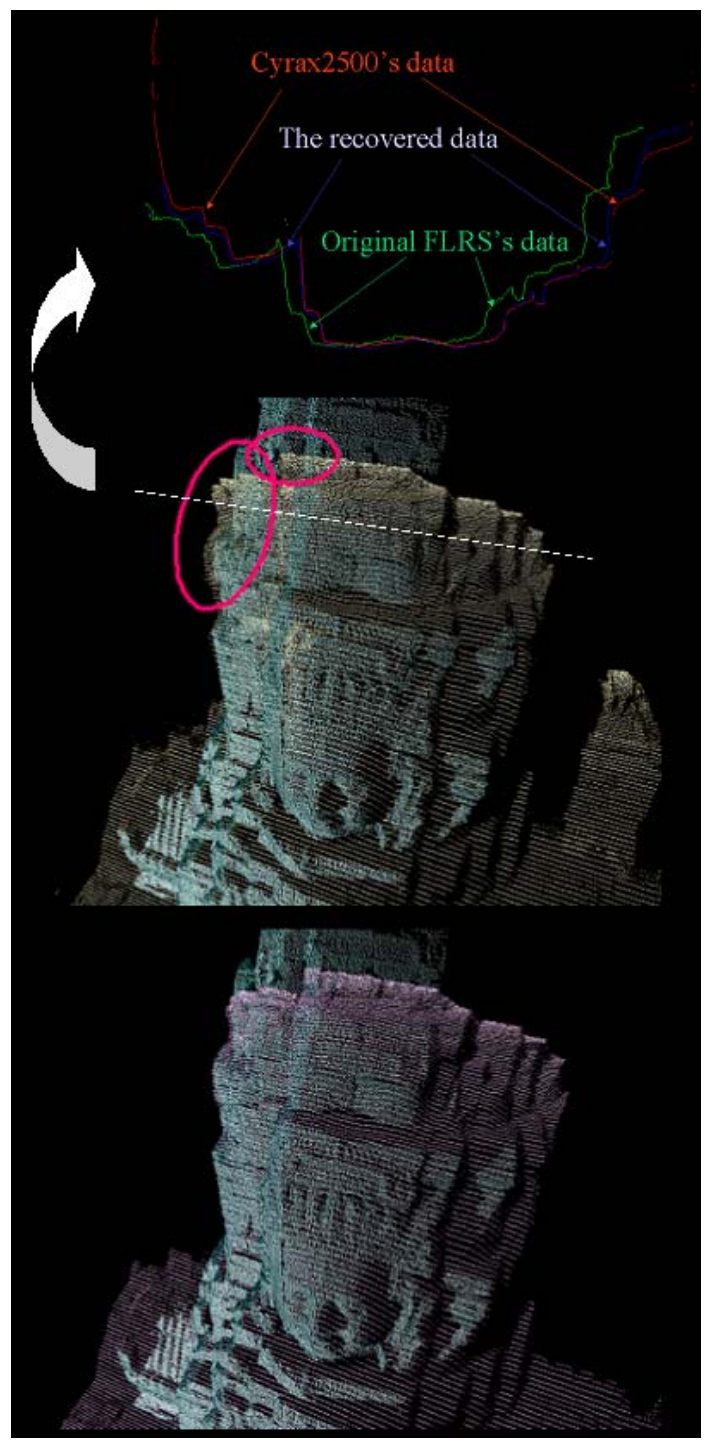

Fig. 3. Range data before and after the recovery process: The middle figure illustrates the registration of the original data from the FLRS (white) and the non-distorted data obtained by the fixed Cyrax 2500 (blue). Two shapes do not coincide, especially at the areas of ellipses in the figure. On the other hand, the recovered shape in the lower figure (pink) is fitted onto the correct data. The upper figure shows the cross section on the white dotted line in the middle figure.

cut off on the white dotted line in the middle figure, also shows the effectiveness.

Another figure, Figure 4, shows the effectiveness of the method. That figure indicates the point-to-point distances between Cyrax2500's data and the recovered data. The left image shows a comparison between the Cyrax2500's and the original distorted data, while the right shows a comparison between the Cyrax2500's and the recovered data. The region where the distances between them are less than $6.0 \mathrm{~cm}$ is colored green(in this study, we set the threshold at $6.0 \mathrm{~cm}$ ). On the other hand the area where the distances are further than $6.0 \mathrm{~cm}$ is displayed in blue. At a glance, the green region

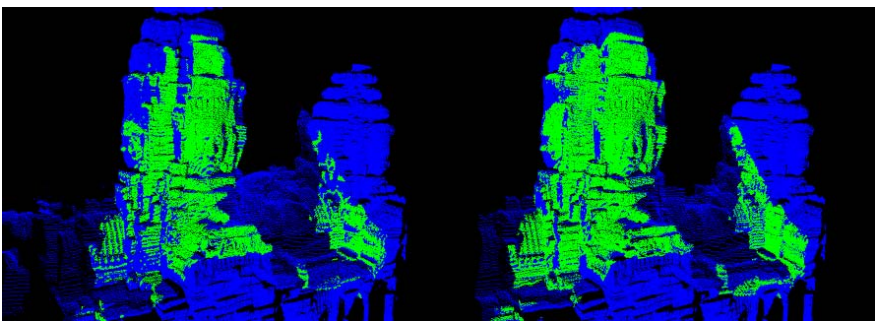

Fig. 4. The comparison between the Cyrax 2500's (the correct data) and the original distorted data (left), and that between the Cyrax 2500 's and the recovered data: the green region indicates where the distance of two shapes is less than $6.0 \mathrm{~cm}$, that is, two shapes coincide in this region. Note that the green region is expanded after the refinement in the right figure.

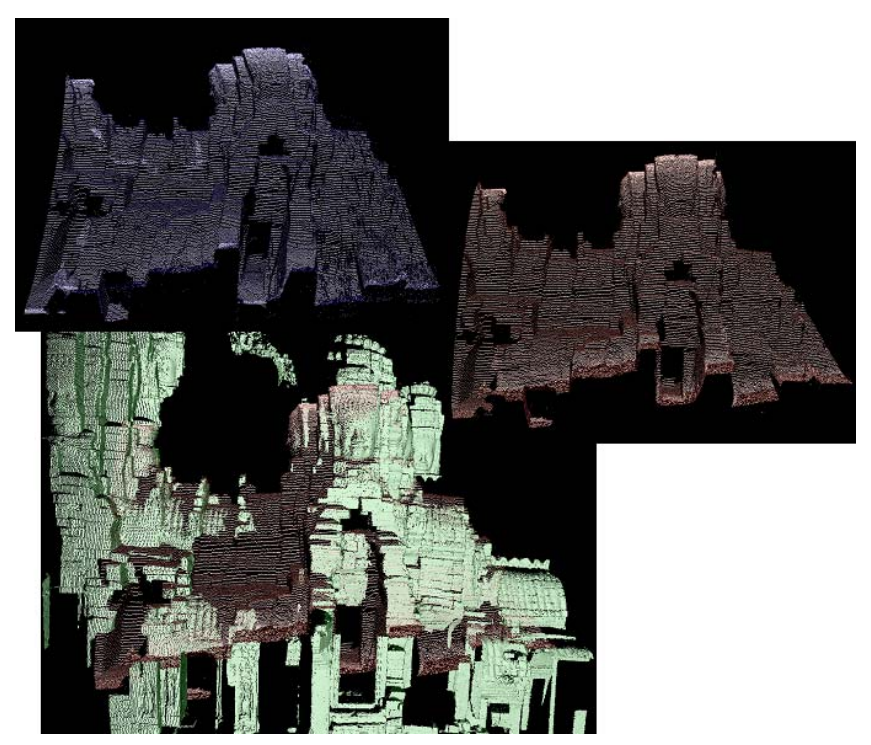

Fig. 5. Another data set by the FLRS: in this case, the original range data are distorted widely (upper left). The recovered shapes are shown in the upper right and the result of registration with Cyrax 2500 's data is shown in the lower.

is clearly expanded by the recovery algorithm. Taking account of the fact the Cyrax 2500 could not measure the upper part of the temple because of occlusions, the method could recover the $3 \mathrm{D}$ shape correctly.

Above data are in the case of that the balloon's motion is rather moderate. Figure 5 shows other data, which are obtained by the balloon in wide motion. One can see that the range data are distorted widely (the upper left image in Fig.5). In this case, we can recover the shape without incident (the upper right). Aligning it with the Cyrax2500's data, the recovered shape is well-fitted with the correct data (the lower image in Fig.5).

\section{CONClusions}

We have presented a method for estimating camera motion. In our framework, we move not only a video camera but also a range sensor. Without any physical sensors such as gyros and GPS, we try to recover the shape of an object and the camera 


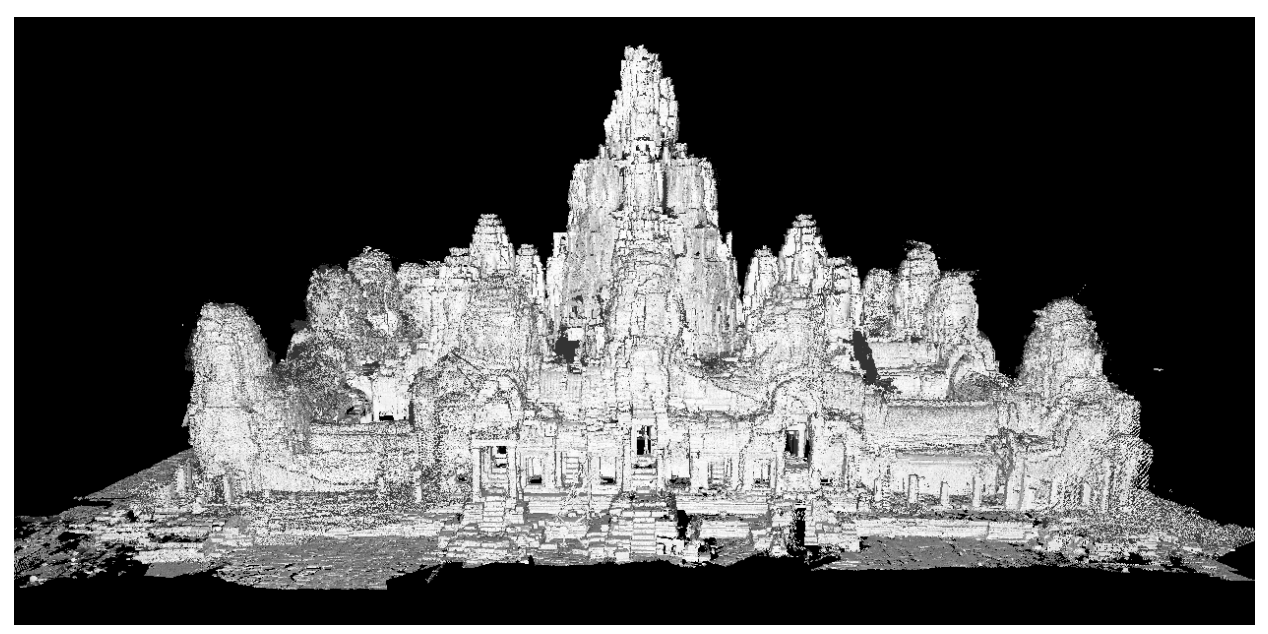

Fig. 6. The overall data of the Bayon Temple

motion by using only an image sequence and the distorted range data. Correct estimation is essential to recover the object shape since, in our FLRS system, a range sensor swings during the measurement process. Using a video sequence on the FLRS and the distorted range data, we have estimated the camera motion. At first, the perspective factorization derived the initial value for the estimation. Next, we solved a nonlinear optimal problem under three constraints. In this process, we utilized distorted data which had been formerly sloughed. Finally, by using estimated camera motion parameters, we recovered the shapes (Figure 6 shows the overall 3D data of the Bayon Temple). This framework can be generally applied to a framework in which a range sensor moves during the scanning process, and is not limited to our FLRS.

We achieved high accuracy recovery of the distorted range data. Our FLRS is expected to be effective for measurement of large scale objects. Therefore, we have to improve the precision obtained by this algorithm.

On the other hand, several problems remain in this method. For example, in the present system, all tracked interest points must be visible throughout a sequence. The tracking algorithm is so simple that it would be rather difficult to track points when balloon's movement is violent.

\section{ACKNOWLEDGMENT}

This work was supported in part by Ministry of Education, Culture, Sports, Science and Technology, under the program, "Development of High Fidelity Digitization Software for Large-Scale and Intangible Cultural Assets", Japan Science and Technology Corporation(JST) and Research Fellowships of Japan Society for the Promotion of Science(JSPS) for Young Scientists. Scanning the Bayon Temple was conducted jointly with Japanese Government Team for Safeguarding Angkor(JSA) and JST.

\section{REFERENCES}

[1] P.J.Besl and N.D.McKay, "A method for registration of 3-D shapes," IEEE Trans. on PAMI, vol.14, pp.239-256, 1992.
[2] J.Costeira and T.Kanade, "A multi-body factorization method for motion analysis," Proc. of ICCV, pp.1071-1076, 1995.

[3] M.Han and T.Kanade, "Perspective factorization methods for Euclidean reconstruction," CMU-RI-TR-99-22, 1999.

[4] Y.Hirota, T.Masuda, R.Kurazume, K.Ogawara, K.Hasegawa and K.Ikeuchi, "Flying Laser Range Finder and its data registration algorithm," Proc. of ICRA, pp.3155-3160, 2004.

[5] D. A. Jacobs, The State of the Art in Numerical Analysis, London: Academic Press, 1977.

[6] D.G.Lowe, "Distinctive image features from scale-invariant keypoints," International Journal of Computer Vision, Vol.60, No.2, pp.91-110, 2004.

[7] D.Miyazaki, T.Oishi, T.Nishikawa, R.Sagawa, K.Nishino, T.Tomomatsu, Y.Yakase and K.Ikeuchi "The great buddha project: Modelling cultural heritage through observation" Proc. of VSMM, pp.138-145, 2000.

[8] T.Morita and T.Kanade, "A sequential factorization method for recovering shape and motion from image streams," IEEE Trans. on PAMI, vol.19, No.8, pp.858-867, 1997.

[9] C.Poelmann and T.Kanade "A paraperspective factorization method for shape and motion recovery," IEEE Trans. on PAMI, vol.19, No.3, pp.206-218, 1997.

[10] E. Polak, Computational Methods in Optimization, New York: Academic Press, 1971.

[11] J. Stoer and R.Bulirsh, Introduction to Numerical Analysis, New York: Springer-Verlag, 1980.

[12] S.Thrun, M.Diel and D.Haehnel, "Scan alignment and 3-D surface modeling with a helicopter platform," The 4th International Conference on Field and Service Robotics, 2003.

[13] C.Tomasi and T.Kanade, "Shape and motion from image streams under orthography: a factorization method," International Journal of Computer Vision, Vol. 9, No.2, pp. 137-154, 1992.

[14] J.Visnovcova, L.Zhang and A.Gruen, "Generating a 3D model of a bayon tower using non-metric imagery," Proc. of the International Workshop Recreating the Past -Visualization and Animation of Cultural Heritage, 2001.

[15] Z.Zhang, "Iterative point matching for registration of free-form curves and surfaces," International Journal of Computer Vision, Vol.13, pp.119-152, 1994. 